\title{
Acquired hepatocerebral degeneration in a patient with hepatitis $B$ and hepatitis delta virus coinfection
}

\author{
Viviane de Carvalho ${ }^{[1]}$, Dessana Francis Chehuan ${ }^{[1]}$ and Marcia Melo Damian ${ }^{[1]}$
}

[1]. Departamento de Ensino e Pós-Graduação, Fundação de Medicina Tropical Heitor Vieira Dourado, Manaus, AM, Brasil.

\begin{abstract}
Acquired hepatocerebral degeneration is a neurological syndrome with typical clinical (extrapyramidal and neuropsychiatric) symptoms and brain magnetic resonance imaging findings (high T1 signal in the globus pallidus). It occurs mainly in patients with advanced liver disease, such as in patients co-infected with hepatitis B virus (HBV) and hepatitis delta virus (HDV). However, there are no reports relating HBV/HDV coinfection and acquired hepatocerebral degeneration. This report presents the case of a 49-year-old woman with characteristics of acquired hepatocerebral degeneration and liver cirrhosis due to HBV/HDV coinfection, and presents the main theories of the physiopathology of this condition.
\end{abstract}

Keywords: Acquired hepatocerebral degeneration. Hepatitis B virus. Hepatitis delta virus.

\section{INTRODUCTION}

Globally, approximately 400 million people have chronic hepatitis B virus (HBV) infection and approximately 24 million have chronic hepatitis delta virus (HDV) infection. In the Brazilian Amazon region, $41.9 \%$ of HBV surface antigen (HBsAg) positive individuals are HDV coinfected ${ }^{1}$. HBV/HDV coinfection accelerates progression to chronic liver disease, cirrhosis, and hepatocellular carcinoma ${ }^{2}$.

Advanced liver disease frequently causes neurological manifestations, including hepatic encephalopathy, hepatic myelopathy, and acquired hepatocerebral degeneration $(\mathrm{AHD})^{3}$. AHD, which is underdiagnosed, is a rare and usually irreversible neurological syndrome (some cases can be reversed by liver transplantation) whose pathophysiology is poorly understood. It usually presents with extrapyramidal signs (ataxia, Parkinsonism, chorea, athetosis, ballism, and cerebellar signs) and varying degrees of neuropsychiatric and cognitive impairments ${ }^{4}$. It has been described in patients with cirrhosis due to $\mathrm{HBV}$ infection, hepatitis $\mathrm{C}$ virus (HCV) infection, or alcoholism $^{3-5}$. However, there are no case reports relating HBV/ HBD coinfection with AHD. The objective of this report is to present the case of a patient with clinical and radiological characteristics of AHD and chronic liver disease due to HBV/ HDV coinfection.

Corresponding author: Dra. Viviane de Carvalho.

e-mail: vivicarvalhofono@yahoo.com.br

Received 9 November 2016

Accepted 6 March 2017

\section{CASE REPORT}

The patient, a 49 year old married woman originally from Manacapuru in the State of Amazonas, Brazil, who worked as an agriculturist, had a history of chronic liver disease, diagnosed in 2014. The chronic liver disease was due to HBV and HDV infection. Her serology results were: HbsAg positive, HBV surface antibody (anti-HBs) negative, total HBV core antibody (anti-HBc) positive, hepatitis B virus-deoxyribonucleic acid (HBV-DNA) negative, total HDV antibody (anti-HDV) positive, and hepatitis delta virus-ribonucleic acid (HDV-RNA) positive. Quantitative determination of serum HDV-RNA and HBV-DNA was performed using real-time reverse transcription polymerase chain reaction assays, available, respectively, in private laboratories and governmental services in Manaus, State of Amazonas. The HDV-RNA viral load was 106.401 copies $/ \mathrm{mL}$, the HBV-DNA viral load was undetectable $(<1 \mathrm{UI} / \mathrm{mL}$ $[<3.41$ copies $/ \mathrm{mL}])$. The patient denied alcoholism and the results of serological tests for $\mathrm{HCV}$ and rheumatic markers were negative. She was followed up at the Service of Hepatology on an outpatient basis and was treated with entecavir.

In May 2015, the patient began to experience tremors in her extremities; this was associated with bradykinesia and postural instability. She consulted a neurologist who made a clinical diagnosis of Parkinson's disease. No familial neurological diseases were reported. She was started on levodopa, which she took for 2 months. This treatment was abandoned after another specialist whose opinion was sought attributed the motor disorders to hepatic encephalopathy, despite the absence of impaired consciousness. In July 2016, her family brought her to the emergency service with a 5-day history of abrupt 
involuntary corporal movements that followed a pattern different to her habitual movements, and were objectively associated with vertigo

The initial neurological examination revealed a Glasgow Coma Scale score of 15. At rest, swinging movements of the limbs and associated facial grimacing were observed. Her cranial nerve function was intact. She had normal motor function and preserved sensation in all extremities. Haloperidol (10mg) was initiated; after 2 days, the choreiform movements had stopped. The patient then showed features of bradykinesia, hypomimia, hypophonia, postural rigidity and instability, diadochokinesia, and a resting tremor. Laboratory examination revealed a normal hematocrit, a platelet count of $88 \times 10^{9} / \mathrm{L}$, prothrombin time of $15.3 \mathrm{~s}$; international normalized ratio of 1.29 , serum total bilirubin of $1.84 \mathrm{mg} / \mathrm{dL}(31.47 \mu \mathrm{mol} / \mathrm{L})$, direct bilirubin $0.51 \mathrm{mg} / \mathrm{dL}(8.72 \mu \mathrm{mol} / \mathrm{L})$, serum glutamic oxaloacetic transaminase $116 \mathrm{U} / \mathrm{L}$, serum glutamic pyruvate transaminase $36 \mathrm{U} / \mathrm{L}$, alkaline phosphatase $285 \mathrm{U} / \mathrm{L}$, gamma-glutamyl transpeptidase $65 \mathrm{IU} / \mathrm{L}$, and albumin $35 \mathrm{~g} / \mathrm{L}$. Serology results for human immunodeficiency virus and HCV were negative. The Child-Pugh score was 6 .

On abdominal ultrasound examination, the liver contours were lobulated with blunt edges, the right lobe of the liver was reduced in size, and the parenchymal echotexture was characteristic of chronic liver disease. Magnetic resonance imaging (MRI) of her brain showed symmetrical high signal intensity in the globus pallidus on T1-weighted images and symmetrical high signal intensities in the caudate nucleus, putamen, globus pallidus, middle cerebellar peduncle, cerebellar hemispheres, and thalamus on T2-weighted images, bilaterally (Figure 1, Figure 2 and 3). After reviewing the literature and clinically discussing the case, the most likely diagnosis was hypothesized to be AHD. Levodopa was prescribed and the dose titrated, with partial control of the symptoms.

\section{DISCUSSION}

HDV infection is considered the most serious form of viral hepatitis. $\mathrm{HDV}$ is a hepatotropic RNA virus that requires $\mathrm{HBsAg}$ in order to assemble new viral particles ${ }^{6}$. Histological examination of the HDV-infected liver demonstrates hepatocellular necrosis and inflammation; these findings are indistinguishable from those caused by other acute or chronic liver diseases ${ }^{7}$. Probably because of that, the literature shows that the development of AHD fundamentally depends on the presence of advanced chronic liver disease, regardless of its etiology.

The first manifestations of AHD are reported to occur at 50-60 years of age. In our clinical case, these were noticed at least 5 years earlier, showing that HBV/HDV coinfection might accelerate not only the progression of chronic liver disease but also the development of AHD. The literature shows that in adult carriers of HBsAg who are coinfected with HDV, the period of progression to chronicity is 2-6 years, whereas in children, chronicity occurs more quickly. HDV also has a remarkable ability to dominate and suppress other viral agents (such as $\mathrm{HBV}$ and HCV), especially in patients with chronic hepatitis and cirrhosis ${ }^{2}$, as shown in the case presented.

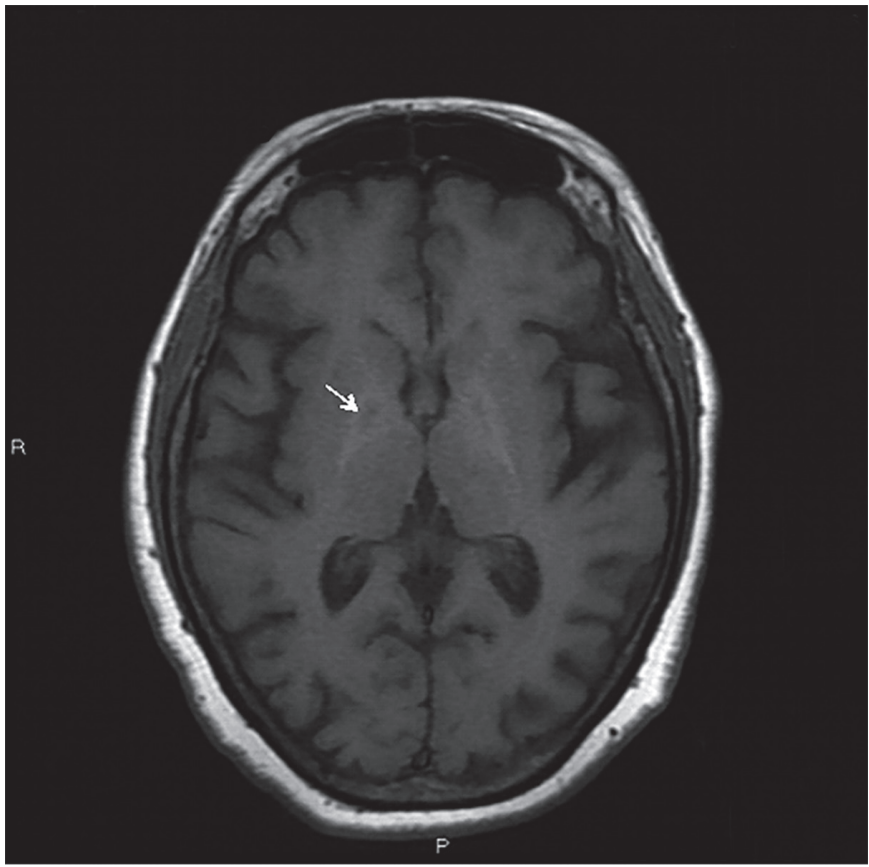

FIGURE 1 - Axial T1-weighted magnetic resonance image demonstrating symmetrical, increased T1 signals in the globus pallidus bilaterally (white narrow).

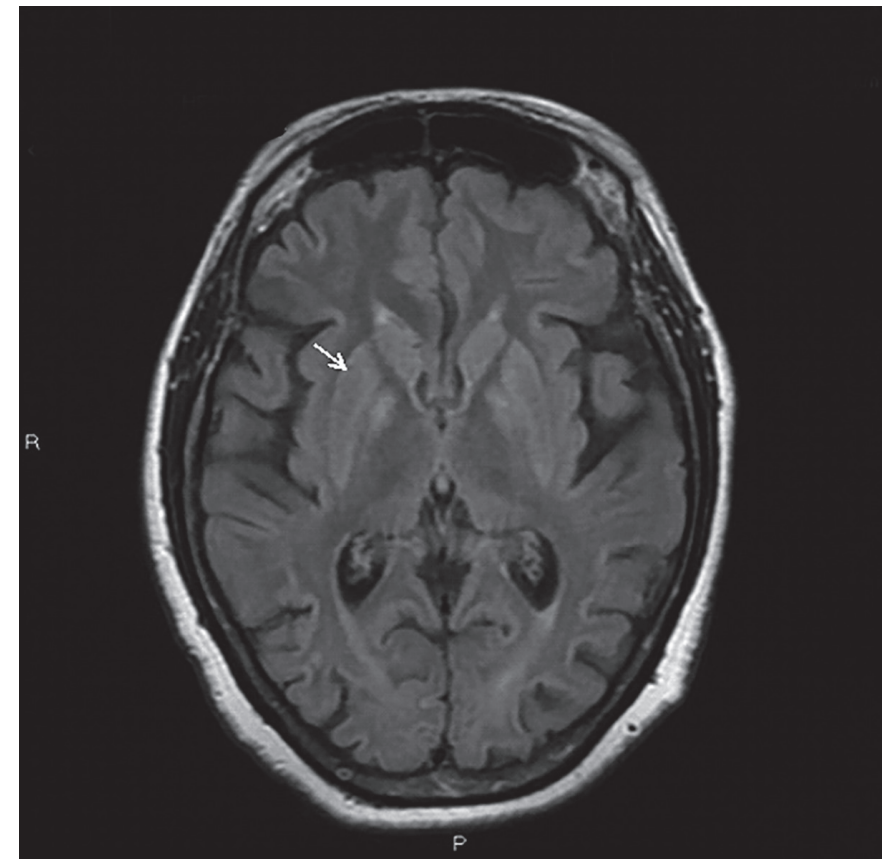

FIGURE 2 - Axial flair T2-weighted magnetic resonance image showing increased signals in the caudate nucleus, putamen (white narrow), and globus pallidus bilaterally.

The pathogenesis of ADH has not yet been clearly elucidated. One hypothesis suggests that the presence of portosystemic shunts is a pre-condition to developing $\mathrm{ADH}^{3,4,5}$. This supposition is made because deviation of portal blood to the systemic circulation would result in the passage of neurotoxic substances, such as amino acids, ammonia, and heavy metals including manganese, that have not been removed by the 


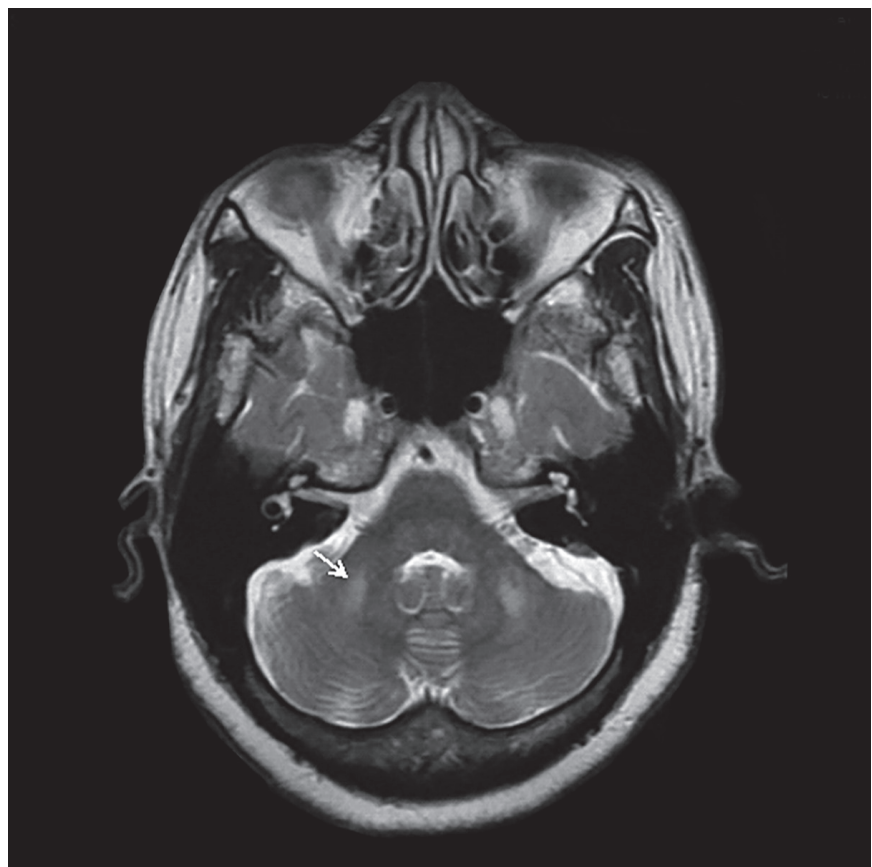

FIGURE 3 - Axial T2-weighted magnetic resonance image demonstrating increased T2 signals in the cerebellar cortex and middle cerebellar peduncles bilaterally (white narrow).

hepatobiliary system, through the blood brain barrier into the central nervous system $(\mathrm{CNS})^{8-10}$. Accumulation of manganese in the CNS could result in increased levels of glutamate/ glutamine and reduced levels of myo-inositol (found in both hepatic encephalopathy and AHD). These changes exert a selective neurotoxic effect, affecting the integrity of neurons and astrocytes, mainly in the basal nuclei, brainstem, and white matter ${ }^{8}$. In the basal ganglia, manganese might accumulate in the mitochondria of the globus pallidus, thereby damaging the glial cells, as suggested by the diffuse hyperplasia of astrocytes, microcavitation, and zonal necrosis observed ${ }^{5,6,9}$. Moreover, this would also cause cellular loss and gliosis in the caudate nucleus, putamen, and subthalamus nucleus. This hypothesis is supported by observational studies of manganese miners who developed extrapyramidal symptoms; studies in that population showed the same neuropathological characteristics as those described in patients with AHD. In addition, the satisfactory therapeutic response to manganese chelators seen in patients with cirrhosisrelated Parkinsonism further reinforces this theory 9

Manganese also seems to interfere with dopamine metabolism. In a prospective study of 214 patients with liver cirrhosis who were eligible for liver transplantation, 9 demonstrated features of Parkinsonism ${ }^{11}$. Positron emission tomography scanning revealed significant reduction in the availability of the $\mathrm{D} 2$ dopamine receptor in 5 of the 9 patients studied and a significant reduction in the dopamine transporter in 3. These data suggest that the alterations that damage presynaptic and striatal pro-synaptic neurotransmission could be the cause of Parkinsonism, explaining the limited effect of dopaminergic therapy. However, other mechanisms, such as genetic predisposition, could play a role in the increased deposition of manganese in the CNS of patients with cirrhosis, not only of those with Parkinsonism ${ }^{8,11}$. Our hypothesis is that, although not visualized on abdominal ultrasonography, our patient developed a portosystemic shunt. Such shunts are difficult to visualize, as has been reported by other authors.

Basal ganglia hyperintensity demonstrated on T1weighted MRI suggests the diagnosis of AHD. Manganese is a paramagnetic metal that shortens the relaxation time of T1 enough to produce hyperintensity in the globus pallidus; however, the cause is not well established ${ }^{10}$. Such findings were observed in our patient. The cerebellum and the middle cerebellar peduncles are considered areas that are highly vulnerable to metabolic insults due to liver disease. In this clinical case, the patient presented with signs of cerebellar alteration (diadochokinesia and wide-based gait). The MRI also revealed hyperintensity in the cerebellar area on T2-weighted images, a characteristic finding in AHD. Focal abnormalities in the cerebellum have been attributed to spongiform degeneration, with increased water content or myelinolysis; however, these abnormalities can also occur with insults from poisonous metabolites and/or osmotic changes ${ }^{12}$.

There are still controversies about the best treatment for AHD. Ferro et al. ${ }^{9}$ reviewed the literature and found works indicating that 11 of 16 patients were successfully treated with levodopa, while 9 of 13 patients were successfully treated with manganese chelators. They concluded that more data are required to identify the more promising treatment strategy. Our patient began treatment with levodopa and attained partial symptom improvement and clinical stability. She is still followed-up on an outpatient basis.

In conclusion, it is imperative that AHD is included in the differential diagnosis of hepatic encephalopathy and other motor alterations in patients with chronic liver disease, because the clinical evolution, treatment, and prognosis of these conditions differ.

\section{Conflict of interest}

The authors declare that have no conflicts of interest.

\section{REFERENCES}

1. Braga WSM, Castilho MC, Borges FG, Leão JRDT, Martinho ACS, Rodrigues, IS et al. Hepatitis D virus infection in the western Brazilian Amazon - far from a vanishing disease. Rev Soc Bras Med Trop. 2012;45(6):691-95.

2. Siederdissen CHZ, Cornberg M. Management of HBV and HBV/ HDV-associated liver cirrhosis. Visc Med. 2016;32(2):86-94.

3. Huang FZ, Hou X, Zhou TQ, Chen S. Hepatic encephalopathy coexists with acquired chronic hepatocerebral degeneration. Neurosciences. 2015;20(3):277-79.

4. Ferro JM, Oliveira S. Neurologic Manifestations of Gastrointestinal and Liver Diseases. Curr Neurol Neurosci Rep. 2014;14(10):487.

5. Listik C, Machado-Porto GCL, Oliveira MO, Porto FHG. Acquired hepatocerebral degeneration: A case report. Dement Neuropsychol. 2012;6(1):59-63. 
6. Karayiannis P. Hepatitis D virus. Rev Med Virol. 1998;8(1)113-24.

7. Nicolete LDF, Borzacov LMP, Vieira DS, Nicolete R, Salcedo JMV. Correlation between TH1 response standard cytokines as biomarkers in patients with the delta virus in the western Brazilian Amazon. Mem Inst Oswaldo Cruz. 2016;111(4):275-76.

8. Chen Y, Haque M, Yoshida EM. Transient improvement of acquired hepatocerebral degeneration with parkinsonian symptoms after failed liver transplant: Case report and literature review. Exp Clin Transplant. 2011;9(6):363-69.

9. Ferro JM, Viana P, Santos P. Management of neurologic manifestations in patients with liver disease. Curr Treat Options Neurol. 2016;18(8):37.
10. Sureka B, Bansal K, Patidar Y, Rajesh S, Mukund A, Arora A. Neurologic Manifestations of Chronic Liver Disease and Liver Cirrhosis. Curr Probl Diagn Radiol. 2015; 44(5):449-61.

11. Tryc AB, Goldbecker A, Berding G, Rümke S, Afshar K, Shahrezaei $\mathrm{GH}$, et al. Cirrhosis-related Parkinsonism: Prevalence, mechanisms and response to treatments. J Hepatol. 2013;58(4):698-705.

12. Park SA, Heo K. Prominent cerebellar symptoms with unusual magnetic resonance imaging findings in acquired hepatocerebral degeneration. Arch Neurol. 2004; 61(9):1458-60. 